\title{
A note on a three point boundary value problem for second order differential inclusions
}

Mouffak Benchohra and Sotiris K. Ntouyas 
Mathematical Notes, Miskolc, Vol. 2., No. 1., (2001), pp. 39-47

\title{
A NOTE ON A THREE POINT BOUNDARY VALUE PROBLEM FOR SECOND ORDER DIFFERENTIAL INCLUSIONS
}

\author{
Mouffak Benchohra \\ Département de Mathématiques, Université de Sidi Bel Abbès \\ BP 89, 22000 Sidi Bel Abbès, Algérie \\ benchohra@yahoo.com \\ Sotiris K. NTOUYAS \\ Department of Mathematics, University of Ioannina \\ 45110 Ioannina, Greece \\ sntouyas@cc.uoi.gr
}

[Received March 6, 2001]

\begin{abstract}
In this paper we investigate the existence of solutions on a compact interval to a three-point boundary value problem for a class of second order differential inclusions. We shall rely on a fixed point theorem for condensing maps due to Martelli.

Mathematical Subject Classification: 34A60, 34B10, 34B15

Keywords: Three point boundary value problems, differential inclusion, existence, fixed point
\end{abstract}

\section{Introduction}

The existence of solutions on compact intervals for three-point boundary value problems for second order differential equations has received much attention in the last decade. We refer for instance to the papers of Boucherif and Bouguima [1], Gupta [2], [3], [4], [5], Gupta et al [6], [7], [8], Gupta and Trofimchuk [7], [8] and Marano [9]. However, to our knowledge, this type of problem has not been studied for the multivalued case.

The study of multi-point boundary value problems for second order ordinary differential equations was initiated by Il'In and Moiseev in [10], [11] motivated by the work of Bitsadze and Samarskii on nonlocal elliptic boundary value problems, 12], [13], [14].

The methods used are usually the topological transversality of Granas or the degree theory methods combined with Wirtinger type inequalities.

In this paper we shall prove a theorem which assures the existence of solutions defined on a compact real interval for the three-point boundary value problem (BVP 
for short) of the second order differential inclusion

$$
\begin{gathered}
y^{\prime \prime} \in F(t, y), \quad t \in J=[0,1] \\
y(0)=0, y(\eta)=y(1),
\end{gathered}
$$

where $F: J \times \mathbf{R} \longrightarrow 2^{\mathbf{R}}$ is a multivalued map with compact convex values and $\eta \in(0,1)$.

The method we are going to use is to reduce the existence of solutions to problem (1.1)-(1.2) to the search for fixed points of a suitable multivalued map on the Banach space $C(J, \mathbf{R})$. In order to prove the existence of fixed points, we shall rely on a fixed point theorem for condensing maps due to Martelli [15].

\section{Preliminaries}

In this section, we introduce notations, definitions, and preliminary facts from multivalued analysis which are used throughout this paper.

Let $(X,\|\cdot\|)$ be a Banach space. A multivalued map $G: X \longrightarrow 2^{X}$ is convex (closed) valued if $G(x)$ is convex (closed) for all $x \in X$. $G$ is bounded on bounded sets if $G(B)=\cup_{x \in B} G(x)$ is bounded in $X$ for any bounded set $B$ of $X$ (i.e. $\sup _{x \in B}\{\sup \{\|y\|: y \in G(x)\}\}<\infty$ ).

$G$ is called upper semicontinuous (u.s.c.) on $X$ if for each $x_{*} \in X$ the set $G\left(x_{*}\right)$ is a nonempty, closed subset of $X$, and if for each open set $B$ of $X$ containing $G\left(x_{*}\right)$, there exists an open neighbourhood $V$ of $x_{*}$ such that $G(V) \subseteq B$.

$G$ is said to be completely continuous if $G(B)$ is relatively compact for every bounded subset $B \subseteq X$.

If the multivalued map $G$ is completely continuous with nonempty compact values, then $G$ is u.s.c. if and only if $G$ has a closed graph (i.e. $x_{n} \longrightarrow x_{*}, y_{n} \longrightarrow y_{*}, y_{n} \in$ $G x_{n}$ imply $\left.y_{*} \in G x_{*}\right)$.

$G$ has a fixed point if there is $x \in X$ such that $x \in G x$.

In the following $C C(X)$ denotes the set of all nonempty compact and convex subsets of $X$.

A multivalued map $G: J \longrightarrow C C(E)$ is said to be measurable if for each $x \in X$ the function $Y: J \longrightarrow \mathbf{R}$ defined by

$$
Y(t)=d(x, G(t))=\inf \{|x-z|: z \in G(t)\}
$$

is measurable.

Definition 1 A multivalued map $F: J \times \mathbf{R} \longrightarrow 2^{\mathbf{R}}$ is said to be an $L^{1}$-Carathéodory if

(i) $t \longmapsto F(t, y)$ is measurable for each $y \in \mathbf{R}$;

(ii) $y \longmapsto F(t, y)$ is upper semicontinuous for almost all $t \in J$; 
(iii) For each $k>0$, there exists $h_{k} \in L^{1}\left(J, \mathbf{R}_{+}\right)$such that

$$
\|F(t, y)\|=\sup \{\|v\|: v \in F(t, y)\} \leq h_{k}(t) \text { for all }|y| \leq k
$$

and for almost all $t \in J$.

An upper semi-continuous map $G: X \longrightarrow 2^{X}$ is said to be condensing if for any subset $B \subseteq X$ with $\alpha(B) \neq 0$, we have $\alpha(G(B))<\alpha(B)$, where $\alpha$ denotes the Kuratowski measure of noncompactness. For properties of the Kuratowski measure, we refer to Banas and Goebel [16].

We remark that a completely continuous multivalued map is the easiest example of a condensing map. For more details on multivalued maps see the books by Deimling [17] and $\mathrm{Hu}$ and Papageorgiou [18].

We will need the following hypotheses:

(H1) $F: J \times \mathbf{R} \longrightarrow C C(\mathbf{R})$ is an $L^{1}$ - Carathéodory multivalued map.

(H2) There exists a function $H \in L^{1}\left(J, \mathbf{R}_{+}\right)$such that

$$
\|F(t, y)\|:=\sup \{\|v\| \in F(t, y)\} \leq H(t) \text { for almost all } t \in J \text { and all } y \in \mathbf{R} \text {. }
$$

Definition 2 A function $y: J \longrightarrow \mathbf{R}$ is called a solution for the BVP (1.1)-(1.2) if $y$ and its first derivative are absolutely continuous and $y^{\prime \prime}$ (which exists almost everywhere) satisfies the differential inclusion (1.1) a.e. on J and the condition (1.2).

For the multivalued map $F$ and for each $y \in C(J, \mathbf{R})$ we define $S_{F, y}^{1}$ by

$$
S_{F, y}^{1}=\left\{v \in L^{1}(J, \mathbf{R}): v(t) \in F(t, y(t)) \text { for a.e. } t \in J\right\} \text {. }
$$

Our considerations are based on the following lemmas.

[19]. Let $I$ be a compact real interval and $X$ be a Banach space. Let $F$ be a multivalued map satisfying (H1) and $S_{F, y}^{1} \neq \emptyset$ for any $y \in C(J, X)$. Let $\Gamma$ be a linear continuous mapping from $L^{1}(I, X)$ to $C(I, X)$, then the operator

$$
\Gamma \circ S_{F}: C(I, X) \longrightarrow C C(C(I, X)), y \longmapsto\left(\Gamma \circ S_{F}\right)(y):=\Gamma\left(S_{F, y}\right)
$$

is a closed graph operator in $C(I, X) \times C(I, X)$.

Lemma 3 [15]. Let $X$ be a Banach space and $N: X \longrightarrow C C(X)$ be a condensing map. If the set

$$
\Omega:=\{y \in X: \lambda y \in N(y) \text { for some } \lambda>1\}
$$

is bounded, then $N$ has a fixed point. 


\section{Main Result}

Now, we are able to state and prove our main theorem.

Theorem 4 Assume that hypotheses (H1)-(H2) hold. Then the BVP (1.1)-(1.2) has at least one solution on $J$.

Proof. Let $C(J, \mathbf{R})$ be the Banach space provided with the norm

$$
\|y\|_{\infty}:=\sup \{\|y(t)\|: t \in J\}, \text { for } y \in C(J, \mathbf{R}) .
$$

Transform the problem into a fixed point problem. Consider the multivalued map, $N: C(J, \mathbf{R}) \longrightarrow 2^{C(J, \mathbf{R})}$ defined by

$$
\begin{array}{r}
N(y)=\left\{h \in C(J, \mathbf{R}): h(t)=\int_{0}^{t}(t-s) g(s) \mathrm{d} s+\frac{t}{1-\eta} \int_{0}^{\eta}(\eta-s) g(s) \mathrm{d} s\right. \\
\left.-\frac{t}{1-\eta} \int_{0}^{1}(1-s) g(s) \mathrm{d} s\right\},
\end{array}
$$

where

$$
g \in S_{F, y}=\left\{g \in L^{1}(J, \mathbf{R}): g(t) \in F(t, y(t)) \text { for a.e. } t \in J\right\}
$$

Remark 5 (i) It is clear that the fixed points of $N$ are solutions to (1.1)-(1.2).

(ii) For each $y \in C(J, \mathbf{R})$ the set $S_{F, y}$ is nonempty (see Lasota and Opial [19]).

We shall show that $N$ satisfies the assumptions of Lemma 3 . The proof will be given in several steps.

Step 1: $N(y)$ is convex for each $y \in C(J, \mathbf{R})$.

Indeed, if $h_{1}, h_{2}$ belong to $N(y)$, then there exist $g_{1}, g_{2} \in S_{F, y}$ such that for each $t \in J$ we have

$h_{i}(t)=\int_{0}^{t}(t-s) g_{i}(s) \mathrm{d} s+\frac{t}{1-\eta} \int_{0}^{\eta}(\eta-s) g_{i}(s) \mathrm{d} s-\frac{t}{1-\eta} \int_{0}^{1}(1-s) g_{i}(s) \mathrm{d} s, \quad i=1,2$.

Let $0 \leq \alpha \leq 1$. Then for each $t \in J$ we have

$$
\begin{aligned}
& \left(\alpha h_{1}+(1-\alpha) h_{2}\right)(t)=\int_{0}^{t}(t-s)\left[\alpha g_{1}(s)+(1-\alpha) g_{2}(s)\right] \mathrm{d} s \\
+ & \frac{t}{1-\eta} \int_{0}^{\eta}(\eta-s)\left[\alpha g_{1}(s)+(1-\alpha) g_{2}(s)\right] \mathrm{d} s-\frac{t}{1-\eta} \int_{0}^{1}(1-s)\left[\alpha g_{1}(s)+(1-\alpha) g_{2}(s)\right] \mathrm{d} s .
\end{aligned}
$$

Since $S_{F, y}$ is convex (because $F$ has convex values), then

$$
\alpha h_{1}+(1-\alpha) h_{2} \in N(y) .
$$


Step 2: $N$ is bounded on bounded sets of $C(J, \mathbf{R})$.

Indeed, it is enough to show that there exists a positive constant $c$ such that for each $h \in N(y), y \in B_{r}=\left\{y \in C(J, \mathbf{R}):\|y\|_{\infty} \leq r\right\}$ one has $\|h\|_{\infty} \leq c$.

If $h \in N(y)$, then there exists $g \in S_{F, y}$ such that for each $t \in J$ we have

$$
h(t)=\int_{0}^{t}(t-s) g(s) \mathrm{d} s+\frac{t}{1-\eta} \int_{0}^{\eta}(\eta-s) g(s) \mathrm{d} s-\frac{t}{1-\eta} \int_{0}^{1}(1-s) g(s) \mathrm{d} s .
$$

By (H1) we have for each $t \in J$ that

$$
\|h(t)\| \leq \int_{0}^{t} h_{r}(s) \mathrm{d} s+\frac{1}{1-\eta} \int_{0}^{\eta}(\eta-s) h_{r}(s) \mathrm{d} s+\frac{1}{1-\eta} \int_{0}^{1}(1-s) h_{r}(s) \mathrm{d} s
$$

Then

$$
|h|_{\infty} \leq \int_{0}^{1} h_{r}(s) \mathrm{d} s+\frac{1}{1-\eta} \int_{0}^{\eta}(\eta-s) h_{r}(s) \mathrm{d} s+\frac{1}{1-\eta} \int_{0}^{1}(1-s) h_{r}(s) \mathrm{d} s=c .
$$

Step 3: $N$ sends bounded sets of $C(J, \mathbf{R})$ into equicontinuous sets.

Let $t_{1}, t_{2} \in J, t_{1}<t_{2}$ and $B_{r}$ be a bounded set of $C(J, \mathbf{R})$. For each $y \in B_{r}$ and $h \in N(y)$, there exists $g \in S_{F, y}$ such that

$$
h(t)=\int_{0}^{t}(t-s) g(s) \mathrm{d} s+\frac{t}{1-\eta} \int_{0}^{\eta}(\eta-s) g(s) \mathrm{d} s-\frac{t}{1-\eta} \int_{0}^{1}(1-s) g(s) \mathrm{d} s, \quad t \in J .
$$

Thus we obtain

$$
\begin{aligned}
\left\|h\left(t_{2}\right)-h\left(t_{1}\right)\right\| & \leq \int_{0}^{t_{2}}\left(t_{2}-s\right)|g(s)| \mathrm{d} s+\int_{t_{1}}^{t_{2}}\left(t_{1}-s\right)\|g(s)\| \mathrm{d} s+ \\
& +\frac{t_{2}-t_{1}}{1-\eta} \int_{0}^{\eta}(\eta-s)\|g(s)\| \mathrm{d} s+\frac{t_{2}-t_{1}}{1-\eta} \int_{0}^{1}(1-s)\|g(s)\| \mathrm{d} s \\
& \leq \int_{0}^{t_{2}}\left(t_{2}-s\right) h_{r}(s) \mathrm{d} s+\int_{t_{1}}^{t_{2}}\left(t_{1}-s\right) h_{r}(s) \mathrm{d} s \\
& +\frac{t_{2}-t_{1}}{1-\eta} \int_{0}^{\eta}(\eta-s) h_{r}(s) \mathrm{d} s+\frac{t_{2}-t_{1}}{1-\eta} \int_{0}^{1}(1-s) h_{r}(s) \mathrm{d} s .
\end{aligned}
$$

As $t_{2} \longrightarrow t_{1}$ the right-hand side of the above inequality tends to zero.

As a consequence of Step 2, Step 3 together with the Ascoli-Arzela theorem we can conclude that $N$ is completely continuous.

Step 4: $N$ has a closed graph.

Let $y_{n} \longrightarrow y_{*}, h_{n} \in N\left(y_{n}\right)$, and $h_{n} \longrightarrow h_{*}$. We shall prove that $h_{*} \in N\left(y_{*}\right)$.

$h_{n} \in N\left(y_{n}\right)$ means that there exists $g_{n} \in S_{F, y_{n}}$ such that

$h_{n}(t)=\int_{0}^{t}(t-s) g_{n}(s) \mathrm{d} s+\frac{t}{1-\eta} \int_{0}^{\eta}(\eta-s) g_{n}(s) \mathrm{d} s-\frac{t}{1-\eta} \int_{0}^{1}(1-s) g_{n}(s) \mathrm{d} s, t \in J$. 
We must prove that there exists $g_{*} \in S_{F, y_{*}}$ such that

$h_{*}(t)=\int_{0}^{t}(t-s) g_{*}(s) \mathrm{d} s+\frac{t}{1-\eta} \int_{0}^{\eta}(\eta-s) g_{*}(s) \mathrm{d} s-\frac{t}{1-\eta} \int_{0}^{1}(1-s) g_{*}(s) \mathrm{d} s, t \in J$.

Now, we consider the linear continuous operator

$$
\Gamma: L^{1}(J, \mathbf{R}) \longrightarrow C(J, \mathbf{R})
$$

$g \longmapsto \Gamma(g)(t)=\int_{0}^{t}(t-s) g(s) \mathrm{d} s+\frac{t}{1-\eta} \int_{0}^{\eta}(\eta-s) g(s) \mathrm{d} s-\frac{t}{1-\eta} \int_{0}^{1}(1-s) g(s) \mathrm{d} s, t \in J$.

From Lemma, it follows that $\Gamma \circ S_{F}$ is a closed graph operator.

Moreover, from the definition of $\Gamma$ we have

$$
h_{n}(t) \in \Gamma\left(S_{F, y_{n}}\right) .
$$

Since $y_{n} \longrightarrow y_{*}$, it follows from Lemma that

$h_{*}(t)=\int_{0}^{t}(t-s) g_{*}(s) \mathrm{d} s+\frac{t}{1-\eta} \int_{0}^{\eta}(\eta-s) g_{*}(s) \mathrm{d} s-\frac{t}{1-\eta} \int_{0}^{1}(1-s) g_{*}(s) \mathrm{d} s, t \in J$

for some $g_{*} \in S_{F, y_{*}}$.

Step 5: The set

$$
\Omega:=\{y \in C(J, \mathbf{R}): \lambda y \in N(y) \text { for some } \lambda>1\}
$$

is bounded.

Let $y \in \Omega$. Then $\lambda y \in N(y)$ for some $\lambda>1$. Thus there exists $g \in S_{F, y}$ such that

$$
\begin{aligned}
y(t)=\lambda^{-1} \int_{0}^{t}(t-s) g(s) \mathrm{d} s+\lambda^{-1} \frac{t}{1-\eta} \int_{0}^{\eta} & (\eta-s) g(s) \mathrm{d} s- \\
& -\lambda^{-1} \frac{t}{1-\eta} \int_{0}^{1}(1-s) g(s) \mathrm{d} s, \quad t \in J .
\end{aligned}
$$

This implies by (H2) that for each $t \in J$ we have

$$
\|y(t)\| \leq \int_{0}^{t}(t-s) H(s) \mathrm{d} s+\frac{1}{1-\eta} \int_{0}^{\eta}(\eta-s) H(s) \mathrm{d} s+\frac{1}{1-\eta} \int_{0}^{1}(1-s) H(s) \mathrm{d} s .
$$

Thus

$\|y\|_{\infty} \leq \int_{0}^{1}(1-s) H(s) \mathrm{d} s+\frac{1}{1-\eta} \int_{0}^{\eta}(\eta-s) H(s) \mathrm{d} s+\frac{1}{1-\eta} \int_{0}^{1}(1-s) H(s) \mathrm{d} s:=K$.

This shows that $\Omega$ is bounded. Set $X:=C(J, \mathbf{R})$. As a consequence of Lemma 3 we deduce that $N$ has a fixed point which is a solution of (1.1)-(1.2) on $J$. 


\section{Concluding Remarks}

Let $a_{i} \in \mathbf{R}$, with all of the $a_{i}^{\prime} s$ having the same sign, $\xi_{i} \in(0,1), i=1,2, \ldots, m-2$, $0<\xi_{1}<\xi_{2}<\ldots<\xi_{m-2}<1$. Consider the following m-point boundary value problem for second order differential inclusions

$$
\begin{aligned}
& y^{\prime \prime}(t) \in F(t, y(t)), \quad t \in[0,1] \\
& y(0)=0, \quad y(1)=\sum_{i=1}^{m-2} a_{i} y\left(\xi_{i}\right) .
\end{aligned}
$$

It is well known (see [10] for example) that if a function $y \in C^{1}$ satisfies the boundary condition (4.2) and all of the $a_{i}, i=1,2, \ldots, m-2$ have the same sign, then there exists $\eta \in\left[\xi_{1}, \xi_{m-2}\right]$, depending on $y \in C^{1}[0,1]$ such that

$$
y(1)=\alpha y(\eta)
$$

with $\alpha=\sum_{i=1}^{m-2} a_{i}$. Accordingly, the problem of the existence of a solution for the BVP (4.1)- (4.2) can be studied via the use of the a priori estimates for the three point BVP

$$
\begin{gathered}
y^{\prime \prime}(t) \in F(t, y(t)), \quad t \in[0,1] \\
y(0)=0, \quad y(1)=\alpha y(\eta),
\end{gathered}
$$

where $\eta \in(0,1)$ is given. We omit the details, since the proof follows the steps of the proof of Theorem 4, with obvious modifications.

It is obvious that the above method can also be applied to other types of m-point BVPs. For example for the BVPs

$$
\begin{aligned}
& y^{\prime \prime}(t) \in F(t, y(t)), \quad t \in[0,1] \\
& y^{\prime}(0)=0, \quad y(1)=\sum_{i=1}^{m-2} a_{i} y\left(\xi_{i}\right)
\end{aligned}
$$

or

$$
\begin{aligned}
& y^{\prime \prime}(t) \in F(t, y(t)), \quad t \in[0,1] \\
& y(0)=0, \quad y^{\prime}(1)=\sum_{i=1}^{m-2} a_{i} y^{\prime}\left(\xi_{i}\right)
\end{aligned}
$$

which can be reduced to the following three point BVPs:

$$
\begin{aligned}
& y^{\prime \prime}(t) \in F(t, y(t)), \quad t \in[0,1] \\
& y^{\prime}(0)=0, \quad y(1)=\alpha y(\eta)
\end{aligned}
$$


and

$$
\begin{aligned}
y^{\prime \prime}(t) & \in F(t, y(t)), \quad t \in[0,1] \\
y(0) & =0, \quad y^{\prime}(1)=\alpha y(\eta),
\end{aligned}
$$

respectively.

\section{REFERENCES}

[1] Boucherif, A. and Bouguima, S.M.: Nonlinear second order ordinary differential equations with nonlocal boundary conditions, Communications on Applied Nonlinear Analysis, 5, (2) (1998), 73-85.

[2] Gupta, C.P.: Solvability of a three-point boundary value problem for a second order ordinary differential equation, Journal of Mathematical Analysis and Applications, 168, (1992), 540-551.

[3] Gupta, C.P.: A note on a second order three-point boundary value problem, Journal of Mathematical Analysis and Applications, 186, (1994), 277-281.

[4] Gupta, C.P.: A second order m-point boundary value problem at resonance, Nonlinear Analysis 24, (1994), 1483-1489.

[5] Gupta, C.P.: Solvability of a multi-point boundary value problem at resonance, Results in Mathematics 28, (1995), 270-276.

[6] Gupta, C.P., Ntouyas, S.K. and Tsamatos, P.Ch.: On an m-point boundary value problem for second order ordinary differential equations, Nonlinear Analysis, 23, (1994), 1427-1436.

[7] Gupta, C.P., Ntouyas, S.K. and Tsamatos, P.Ch.: Existence results for m-point boundary value problems, Differential Equations and Dynamical Systems, 2, (1994), 289-298.

[8] Gupta, C.P., Ntouyas, S.K. and Tsamatos, P.Ch.: Solvability of an m-point boundary value problem for second order ordinary differential equations, Journal of Mathematical Analysis and Applications, 189, (1995), 575-584.

[9] Gupta, C.P. and Trofimchuk, S.I.: A sharper condition for the solvability of a threepoint second order boundary value problem, Journal of Mathematical Analysis and Applications, 285, (1997), 586-597.

[10] Gupta, C.P. and Trofimchuk, S.I.: A Wirtinger type inequality and a three-point boundary value problem, Dynamic Systems and Applications, 8, (1999), 127-132.

[11] Marano, S.A.: A remark on a second order three-point boundary value problem, Journal of Mathematical Analysis and Applications, 183, (1994), 518-522.

[12] IL'In, V.A. and Moiseev, E.I.: Nonlocal boundary value problem of the first kind for a Sturm-Liouville operator in its differential and finite difference aspects, Differential Equations, 23(7), (1987), 803-810.

[13] IL'in, V.A. and Moiseev, E.I.: Nonlocal boundary value problem of the second kind for a Sturm-Liouville operator, Differential Equations, 23(8), (1987), 979-987.

[14] Bitsadze, A.V.: On the theory of nonlocal boundary value problem, Soviet Mathematics Doklady, 30(1), (1984), 8-10.

[15] Bitsadze, A.V.: On a class of conditionally solvable nonlocal boundary value problems for harmonic functions, Soviet Mathematics Doklady, 31(1), (1985), 91-94. 
[16] Bitsadze, A.V. and SamarskiI, A.A.: On some simple generalizations of linear elliptic boundary value problem, Soviet Mathematics Doklady, 10(2), (1969), 398-400.

[17] M. Martelli: A Rothe's type theorem for noncompact acyclic-valued map, Bolletino Unione Mathematica Italiana, 11, (1975), 70-76.

[18] Banas, J. and Goebel,K.: Measures of Noncompactness in Banach Spaces, MarcelDekker, New York, 1980.

[19] K. Deimling: Multivalued Differential Equations, Walter de Gruyter, Berlin-New York, 1992.

[20] Hu Sh. and Papageorgiou, N.: Handbook of MultiValued Analysis, Volume I: Theory, Kluwer, Dordrecht, Boston, London, 1997.

[21] Lasota,A. and Opial,Z.: An application of the Kakutani-Ky Fan theorem in the theory of ordinary differential equations, Polonaise des Sciences. Série des Sciences Mathématiques, Astronomiques et Physiques, 13 (1965), 781-786. 